\title{
Combining flame monitoring techniques and support vector ma- chine for the online identification of coal blends*
}

\author{
Hao ZHOU ${ }^{\dagger+1}$, Yuan $\mathrm{LI}^{1}$, Qi TANG ${ }^{1}$, Gang LU², Yong YAN ${ }^{2}$ \\ ( ${ }^{1}$ State Key Laboratory of Clean Energy Utilization, Zhejiang University, Hangzhou, 310027, China) \\ $\left({ }^{2}\right.$ School of Engineering and Digital Arts, University of Kent, Canterbury, Kent CT2 7NT, UK) \\ 'E-mail: zhouhao@zju.edu.cn \\ Received xx xx, 20xx; Revision accepted xx xx, 20xx; Crosschecked xx. xx, 20xx
}

\begin{abstract}
The combustion behavior of two single coals and three coal blends in a $300 \mathrm{~kW}$ coal-fired furnace under variable operating conditions was monitored by a flame monitoring system based on image processing and spectral analysis. A similarity coefficient was defined to analyze the similarity of combustion behavior between two different coal types. A total of 20 flame features, extracted by the flame monitoring system, were ranked by weights of their importance estimated using ReliefF, a feature selection algorithm. The mean of the infrared signal was found to have by far the highest importance weight among the flame features. Support vector machine (SVM) was used to identify the coal types. The number of flame features used to build the SVM model was reduced from 20 to 12 by combining the methods of ReliefF and SVM, and computational precision was guaranteed simultaneously. A threshold was found for the relationship between the error rate and similarity coefficient, which were positively correlated. The success rate decreased with increasing similarity coefficient. The results obtained demonstrate that the system can achieve the online identification of coal blends in industry.
\end{abstract}

Key words: Coal blends, Flame monitoring, Online identification, RelifF, SVM, Similarity

doi:xx.xxxx/jzus.Xxxxxxxx

Document code: A

CLC number: $\mathrm{xxxxx.xx}$

\section{Introduction}

In the last decade, coal burning has provided about $70 \%$ of the energy consumed in China. Coal blends have been regarded as an attractive fuel in coal-fired power plants to minimize fuel costs, improve fuel flexibility and reduce pollutant emissions (Haas et al., 2001). The combustion behavior of a coal blend differs from those of its components. The reactivity of a coal blend is different from that of the blended composition, depending

\footnotetext{
${ }^{\ddagger}$ Corresponding author

* Project supported by the National Basic Research Program of China (2015CB251501) and the Key Technologies R\&D Program of China (2012BAA12B03).

(1) ORCID:
}

C Z Zhejiang University and Springer-Verlag Berlin Heidelberg 2016 mainly on the coal rank and mixing ratio (Peralta et al., 2001; Osorio et al., 2008). There is a need to identify the types of coal blends in coal-fired power plants that will enhance boiler safety, improve combustion efficiency and reduce pollutant emissions. Considerable research has been conducted on the combustion behavior of coal blends, including the ignition process, NOx emissions, burnout behavior and slagging behavior, using thermogravimetric analysis and drop-tube furnaces (Cloke et al., 2002; Biswas et al., 2006; Chi et al., 2010; Moon et al., 2013; Sarkar et al., 2013; Zhou et al., 2015). In a coal-fired power plant, usually multiple coal types are burned, and the blended composition is unknown and even unpredictable after delivery and accumulation in stocks. Online identification of coal blends is desirable for continuous combustion optimization in coal-fired 
boilers, since traditional coal analyzers work off-line and are time-consuming.

With the development of diagnostic methods, significant research has been devoted to monitoring coal combustion behavior (Ballester and García-Armingol, 2010). In recent studies, flame monitoring techniques have generally been applied to extract flame features. A two-color method was applied for the two-dimensional (2D) temperature profile measurement of sooting flames (Huang and Yan, 2000; Huang et al., 2000), and a simple two-color method, in which only colorimetric information is needed, was also proposed (Jiang et al., 2009). A flame monitoring system was established to monitor the $2 \mathrm{D}$ frequency profile of coal flames using image processing and spectral analysis ( $\mathrm{Lu}$ et al., 2006). Moreover, an auto-adaptive edge-detection algorithm was put forward to define flame edges clearly and continuously (Qiu et al., 2012). Because of the strengths of using non-intrusive probes in harsh environments (mainly inside boilers), flame monitoring techniques have been used for many purposes, such as co-firing characterizations (Molcan et al., 2009), prediction of pollution emissions (Wang et al., 2002), and combustion optimization (Huang et al., 2010).

Machine learning algorithms are applied to identify coal types using the flame features extracted by flame monitoring techniques. An approach combining principal component analysis (PCA) and neural network (NN) was proposed for online identification of eight single coals under steady conditions in a $500 \mathrm{~kW}$ coal-fired furnace (Xu et al., 2005). Flame intensity signals were obtained using a three-cell detector covering three spectral bands. SVM combined with PCA and independent component analysis (ICA) was applied to identify eight single coals under normal combustion conditions in a power plant (Tan et al., 2012). A coal identification system based on SVM was used to identify four single coals under variable operating conditions in a $300 \mathrm{~kW}$ coal-fired furnace (Zhou et al., 2014). In addition, a radial basis function (RBF) NN was employed to identify biomass fuels based on flame radical imaging (Li et al., 2015).

Nevertheless, previous studies focused only on the identification of either single coals or biomass. Due to the popularity of coal blends in power plants, a thorough understanding of the identification of coal blends is urgently needed. In this study, a flame monitoring system was applied to acquire both flame images and light intensity signals. A similarity coefficient was defined to investigate the similarity of the combustion behavior between two different coal types using the extracted flame features. The method of ReliefF (Kononenko et al., 1997), a feature selection algorithm, was used to evaluate the weighting factors of all extracted flame features quantitatively. SVM (Cortes and Vapnik, 1995; Chang and Lin, 2011), tackling small sample learning problems very well and exhibiting superior generalization performance in machine learning, was applied to identify coal types. A detailed investigation of the identification of coal blends was conducted based on ReliefF and SVM under variable conditions, in a $300 \mathrm{~kW}$ coal-fired furnace.

\section{Experimental setup}

\subsection{Combustion test facility}

Fig. 1 shows the layout of the $300 \mathrm{~kW}$ coal-fired test facility and the flame monitoring system mounted in the facility. The test facility contains a vertical furnace, a coal feeder, and a swirling burner. The total height of the furnace is $3.95 \mathrm{~m}$, and its internal diameter is $0.35 \mathrm{~m}$. Heat-insulating material was assembled in the furnace to reduce heat dissipation. The coal feeding rate was adjusted between 10 and 45 $\mathrm{kg} / \mathrm{h}$ by an electromotor. The temperature of heated primary air (PA) and secondary air (SA) was about 40 ${ }^{\circ} \mathrm{C}$. PA and SA flow rates were adjusted as required by valves. The flame monitoring system was mounted in the furnace at a distance of $0.244 \mathrm{~m}$ from the swirling burner outlet, which could capture a region of the furnace $0.189 \times 0.151 \mathrm{~m}^{2}$ in area.

\subsection{Flame monitoring system}

A schematic of the flame monitoring system is shown in Fig. 2. The system consists of a $90^{\circ}$ angle lens inside a water-cooling tube, a beam splitter, a three-cell detector, an RGB CMOS camera, a serial device server, a network switch, and a personal computer (PC). The beam splitter is used to divide the flame light into two beams which are captured by the camera and the three-cell detector, respectively. The camera with an RJ45 port can be integrated into a 
network device. The three-cell detector, translating light intensity signals into electrical signals, is designed to capture ultraviolet (UV), visible (VI), and infrared (IR) spectral bands from $240 \mathrm{~nm}$ to $1700 \mathrm{~nm}$. The serial device server is applied to connect the electrical signals translated by the detector to the Ethernet directly. The network switch is used to transmit both flame images and light intensity signals together via the Ethernet. Flame images and light intensity signals are synchronically acquired and processed using the PC with dedicated software. More details of the prototype system are described by Sun et al. (2011). With the introduced serial device and network switch, the signal transmission of the improved system is more stable than that of the prototype system (Sun et al., 2011; Zhou et al., 2014) which is controlled through a remote desktop.

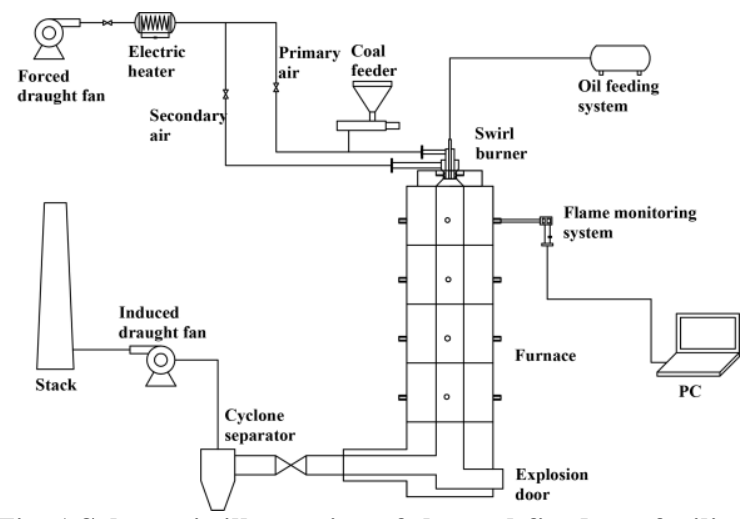

Fig. 1 Schematic illustration of the coal-fired test facility and location of the flame monitoring system

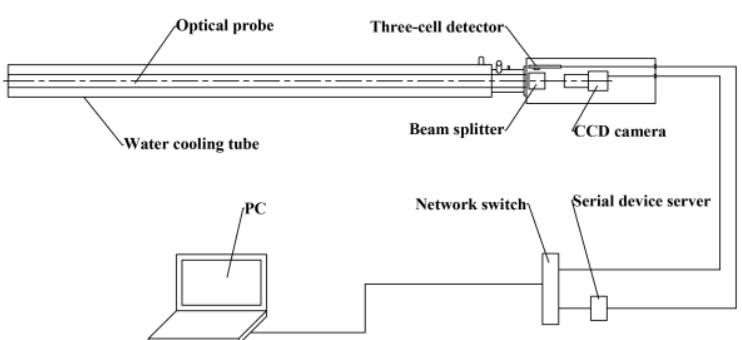

Fig. 2 Schematic of the flame monitoring system

\section{Method}

\subsection{Data acquisition and feature extraction}

The RGB camera resolution was set at $640(\mathrm{H})$ $\times 512$ (V) pixels, the frame rate at $5 \mathrm{fps}$ and the exposure time at $7 \mathrm{~ms}$. The light intensity signals were sampled by the three-cell detector at $256 \mathrm{~Hz}$, and each signal sequence consisted of 1024 data points. Spatial and temporal flame features were characterized.

Fig. 3a shows 20 typical flame images captured by the camera continuously in $4 \mathrm{~s}$. Image processing technique was applied to extract spatial flame features. Spatial flame features include the means and standard deviations of the R, G, and B channels, and the R/G ratios of the flame image. A Guassian filter with a thresholding method was applied to reduce the background noise of the image and improve the signal-to-noise ratio (SNR). The correspond Gaussian function is shown as

$$
G(x, y)=\frac{1}{2 \pi \sigma^{2}} \exp \left(-\frac{\left(x-x_{0}\right)^{2}+\left(y-y_{0}\right)^{2}}{2 \sigma^{2}}\right) .
$$

Then, a morphological filter was used to extract the flame edge (Fig. 3b). A flame zone, defined as the flame region surrounded by the flame edge, was used to extract spatial flame features (Fig. 3c). Means and standard deviations of $\mathrm{R}, \mathrm{G}$, and $\mathrm{B}$ channels are related to the intensity of thermal release, to some extent. The mean and standard deviation of the R/G ratios respond to the temperature of the sooting flame (Jiang et al., 2009).

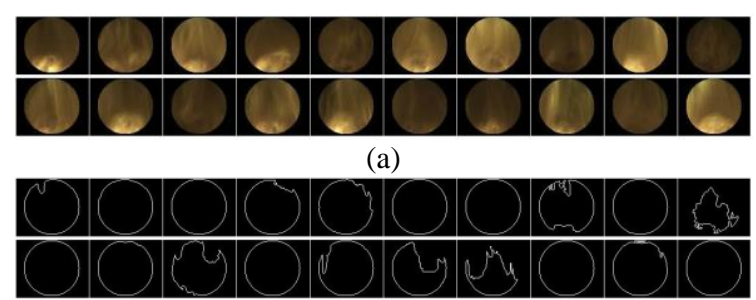

(b)

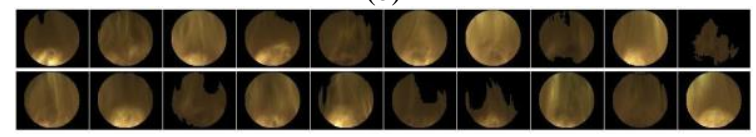

(c)

Fig. 3 Typical flame images and defined flame zones: (a) original flame images ( 20 images); (b) detected flame edges (20 images); (c) defined flame zones ( 20 images)

The means and standard deviations of the R, G, and $\mathrm{B}$ channels, and the $\mathrm{R} / \mathrm{G}$ ratios were defined as follows:

$$
\begin{aligned}
& P_{\text {mean }, i}=\frac{1}{\mathrm{nZ}-1} \sum_{g_{i, j} \in Z} g_{i, j}, \\
& P_{\text {std }, i}=\sqrt{\frac{1}{\mathrm{nZ}-1} \sum_{g_{i, j} \in Z}\left(g_{i, j}-P_{\text {mean }, j}\right)^{2}},
\end{aligned}
$$

where $i=1,2,3$, and 4 denote the R, G, and B channels, and the $R / G$ ratios of the flame image, respectively; 
$P_{\text {mean }, i}$ is the mean of the $i$ th signal; $\mathrm{nZ}$ is the pixel number of the flame zone; $g_{i, j}$ is the $j$ th gray-level or ratio of the $i$ th signal; $Z$ is the defined flame zone; and $P_{\text {std, } i}$ is the standard deviation of the $i$ th signal.

Fig. 4 shows the typical VI and IR signals in time domain. The values of light intensity signals are relative because of the different gain settings of the detector for VI and IR signals. Spectral analysis was used to extract temporal flame features. Temporal flame features include the mean, standard deviation, skewness, kurtosis, fluctuation ratio, and oscillation frequency of the light intensity signal. Three levels of wavelet shrinkage were applied to remove the background noise in the light intensity signal. The mean and standard deviation of the light intensity signal indicate their intensity and variation of thermal release. Skewness and kurtosis reflect their symmetry and steepness. The fluctuation ratio and oscillation frequency are related to the stability of the light intensity signal.

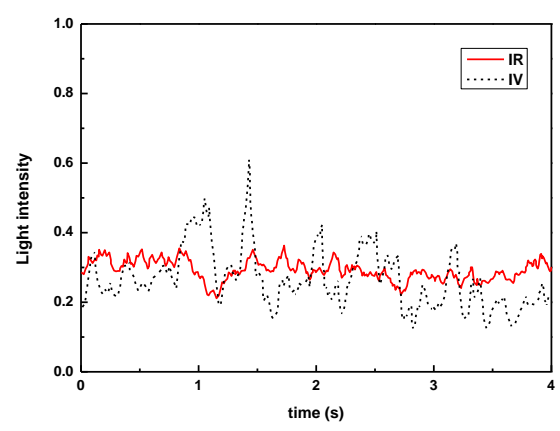

Fig. 4 Typical light intensity signals

The mean, standard deviation, skewness, kurtosis, and fluctuation ratio of the light intensity signal were defined as follows:

$$
\begin{aligned}
& S_{\text {mean }, k}=\frac{1}{N} \sum_{j=1}^{N} s_{k, j}, \\
& S_{\text {std }, k}=\sqrt{\frac{1}{N-1} \sum_{j=1}^{N}\left(s_{k, j}-S_{\text {mean }, k}\right)^{2},} \\
& S_{\text {ske }, k}=\frac{1}{N} \sum_{j=1}^{N}\left(\frac{S_{k, j}-S_{\text {mean }, k}}{S_{\text {std }, k}},\right. \\
& S_{\text {kur }, k}=\frac{1}{N} \sum_{j=1}^{N}\left(\frac{S_{k, j}-S_{\text {mean }, k}}{S_{\text {std }, k}}\right)^{4}, \\
& S_{\text {max }, k}=\max \left(\left[s_{k, 1} \cdots S_{k, N}\right]\right),
\end{aligned}
$$

$$
\begin{aligned}
S_{\min , k} & =\min \left(\left[s_{k, 1} \ldots s_{k, N}\right]\right), \\
S_{\text {flu }, k} & =\frac{S_{\text {max }, k}-S_{\text {min }, k}}{S_{\text {mean }, k}},
\end{aligned}
$$

where $k=1,2$ denote VI and IR signals, respectively; $S_{\text {mean }, k}$ is the mean of the $k$ th signal; $S_{\text {std, } k}$ is the standard deviation of the $k$ th signal; $S_{\text {ske, } k}$ is the skewness of the $k$ th signal; $S_{\mathrm{kur}, k}$ is the kurtosis of the $k$ th signal; $S_{\mathrm{flu}, k}$ is the fluctuation ratio of the $k$ th signal; $N$ is the sampling number; $s_{k, j}$ is the $j$ th signal value of the $k$ th signal; $\left[s_{k, 1} \cdots s_{k, N}\right]$ is the $k$ th signal; $S_{\max , k}$ is the maximum value of the $k$ th signal; and $S_{\min , k}$ is the minimum value of the $k$ th signal.

The oscillation frequency of the light intensity signal was estimated using the Welch power spectral density (PSD) method (Huang and Zhang, 2008) and was defined as

$$
S_{f, k}=\sum_{j=1}^{n} p_{k, j} f_{k, j} / \sum_{j=1}^{n} p_{k, j},
$$

where $k=1,2$ denote VI and IR signals, respectively; $S_{f, k}$ is the oscillation frequency of the $k$ th signal; $f_{k, j}$ is the $j$ th frequency of the $k$ th signal; $p_{k, j}$ is the power density of the $j$ th frequency component; and $n$ is the number of frequency components (Huang and Zhang, 2008).

Only IR and VI signals were used in this study, due to the weakness of the UV signal in the tests. Thus, the total number of flame features was 20 , including spatial and temporal flame features (Table $1)$.

\section{Table 1 List of flame features}

\begin{tabular}{llll}
\hline No. & $\begin{array}{l}\text { Spatial flame } \\
\text { features }\end{array}$ & No. & $\begin{array}{l}\text { Temporal flame } \\
\text { features }\end{array}$ \\
\hline 1 & $P_{\text {mean,R }}$ & 9 & $S_{\text {mean,VI }}$ \\
2 & $P_{\text {mean, }}$ & 10 & $S_{\text {mean,IR }}$ \\
3 & $P_{\text {mean, B }}$ & 11 & $S_{\text {std,VI }}$ \\
4 & $P_{\text {mean,R/G }}$ & 12 & $S_{\text {std,IR }}$ \\
5 & $P_{\text {std, }}$ & 13 & $S_{\text {ske,VI }}$ \\
6 & $P_{\text {std, G }}$ & 14 & $S_{\text {ske,IR }}$ \\
7 & $P_{\text {std, B }}$ & 15 & $S_{\text {kur,VI }}$ \\
8 & $P_{\text {std, R/G }}$ & 16 & $S_{\text {kur,IR }}$ \\
& & 17 & $S_{\text {flu,VI }}$ \\
& & 18 & $S_{\text {flu,IR }}$ \\
& & 19 & $S_{f, \mathrm{VI}}$ \\
& & 20 & $S_{f, \mathrm{IR}}$ \\
\hline
\end{tabular}

Notes: R, R channel; G, G channel; B, B channel; VI, visible signal; IR, infrared signal.

\subsection{Similarity coefficient}

A similarity coefficient was used to analyze the similarity of the combustion behavior between two 
different coal types for each flame feature, and was defined as

$$
\mathrm{Cs}_{\alpha-\beta, i}=\mathrm{Rl}_{i} / \mathrm{Ra}_{i},
$$

where $i$ is the serial number of flame features ranging from 1 to $20, \mathrm{Cs}_{\alpha-\beta, i}$ is the similarity coefficient between coal $\alpha$ and coal $\beta$ in the $i$ th flame feature, $\mathrm{Rl}_{i}$ is the overlap range of the $i$ th flame feature between coal $\alpha$ and coal $\beta$, and $\mathrm{Ra}_{i}$ is the overall range of the $i$ th flame feature between coal $\alpha$ and coal $\beta$. In addition, a weighted average similarity coefficient, $\mathrm{Cs}_{\alpha-\beta, \beta \text { average, was used to estimate the similarity }}$ between coal $\alpha$ and coal $\beta$ for selected flame features, and was defined as

$$
\mathrm{Cs}_{\alpha-\beta, \text { average }}=\frac{\sum_{j \in S} w_{j} \cdot \mathrm{Cs}_{\alpha-\beta, j}}{\sum_{j \in S} w_{j}},
$$

where $j$ is the serial number of the selected flame features, $S$ is the collection of selected flame features, $w_{j}$ (determined by the ReliefF algorithm) is the weighting factor of the $j$ th flame feature, and $\mathrm{Cs}_{\alpha-\beta, j}$ is the similarity coefficient between $\operatorname{coal} \alpha$ and $\operatorname{coal} \beta$ in the $j$ th flame feature.

\subsection{ReliefF algorithm}

The purpose of feature selection is to choose a subset of features which is necessary and sufficient to describe the target concepts (Piramuthu, 2003). Relief is a feature weight based algorithm, which uses the p-dimensional Euclid distance for selecting Near-hits and Near-misses (Kira and Rendell, 1992). Although Relief is noise-tolerant and robust to feature interaction, it is adapted to binary-classification problems only and cannot handle instances of incomplete data. ReliefF, an improved algorithm of Relief, was proposed to deal with multi-class and incomplete datasets (Kononenko et al., 1997).

There are differences in the importance of each flame feature used for coal type identification. The method that models the effects of single flame features (Zhou et al., 2014) is not good for feature selection, and cannot weight the importance of each flame feature quantitatively. In this study, the importance of each flame feature was evaluated quantitatively using the method of ReliefF. This method not only gives the ranking of each flame feature, but also calculates its weighting factor.

\subsection{Support vector machine}

SVM is a machine learning method based on statistical learning theory (SLT) that constructs a hyperplane for classification, regression and other tasks, and is able to tackle small sample learning problems very well (Cortes and Vapnik, 1995; Chang and Lin, 2011). It exhibits superior generalization performance and has strong theoretical motivation in SLT compared with NN algorithms (Leslie et al., 2002). Coal type identification is a classification problem in nature. In this study, support vector classification (SVC) was chosen to model the process and predict the outcome of coal type identification.

Given a set of training data, $\boldsymbol{U}=\left[\left(\boldsymbol{x}_{1}, y_{1}\right),\left(\boldsymbol{x}_{2}\right.\right.$, $\left.\left.y_{2}\right), \cdots\left(\boldsymbol{x}_{n}, y_{n}\right)\right]$, where $\boldsymbol{x}_{i} \in R^{m}$ is a feature vector, $y_{i} \in[-1$, 1] is an indicator vector in two classes, and $f(\boldsymbol{x})=\boldsymbol{w}^{\mathrm{T}} \boldsymbol{\phi}(\boldsymbol{x})+b$ is the linear function, the SVC primal problem (Cortes and Vapnik, 1995) is expressed as

$$
\begin{array}{ll}
\frac{\min }{\boldsymbol{w}, b, \boldsymbol{\xi}} & \left\{\frac{1}{2} \boldsymbol{w}^{\mathrm{T}} \boldsymbol{w}+C \sum_{i=1}^{n} \xi_{i}\right\}, \\
\text { s.t. } \quad & y_{i}\left(\boldsymbol{w}^{\mathrm{T}} \phi\left(\boldsymbol{x}_{i}\right)+b\right) \geq 1-\xi_{i}, \\
& \xi_{i} \geq 0, \quad i=1, \cdots, n,
\end{array}
$$

where $C$ is the regularization parameter, $f\left(\boldsymbol{x}_{i}\right)$ maps $\boldsymbol{x}_{i}$ into a higher-dimensional space, $\boldsymbol{w}$ is the weight vector and $b$ is the bias. In most cases, the vector $\boldsymbol{w}$ is always a high dimensional variable. The corresponding SVC dual problem is defined as

$$
\begin{array}{ll}
\min _{\alpha} & \frac{1}{2} \boldsymbol{\alpha}^{\mathrm{T}} Q \boldsymbol{\alpha}-\boldsymbol{e}^{\mathrm{T}} \boldsymbol{\alpha}, \\
\text { s.t. } & y^{\mathrm{T}} \boldsymbol{\alpha}=0, \\
& 0 \leq \alpha_{i} \leq C, \quad i=1, \cdots, n,
\end{array}
$$

where $\boldsymbol{e}=[1, \cdots 1]_{n}^{\mathrm{T}}, \alpha_{i}$ is the Lagrange multiplier, $\boldsymbol{Q}=\left[y_{i} y_{j} K\left(\boldsymbol{x}_{i}, \boldsymbol{x}_{j}\right)\right]_{n \times n}$ is an n-by-n positive semi-definite matrix, and $K\left(\boldsymbol{x}_{i}, \boldsymbol{x}_{j}\right)=f\left(\boldsymbol{x}_{i}\right)^{T} f\left(\boldsymbol{x}_{j}\right)$ is the kernel function. After the dual problem is solved, the optimal variable $\boldsymbol{w}$ can be expressed as

$$
\boldsymbol{w}=\sum_{i=1}^{n} y_{i} \alpha_{i} \phi\left(\boldsymbol{x}_{i}\right)
$$

The decision function is formulated as

$$
\operatorname{sgn}\left(\boldsymbol{w}^{T} \phi(\boldsymbol{x})+b\right)=\operatorname{sgn}\left(\sum_{i=1}^{n} y_{i} \alpha_{i} K\left(\boldsymbol{x}_{i}, \boldsymbol{x}\right)+b\right)
$$

As the practical problem is nonlinear, a Gaussian RBF is used as the kernel function, resulting in a Hilbert space of infinite dimension. In addition, the one-against-one method (Hsu and Lin, 2002) is 
introduced to solve the multiclass classification problem. In this study, LIBSVM (Chang and Lin, 2011), one of the most popular SVM software packages, was used to identify the coal types.

\subsection{Online identification system}

Fig. 5 illustrates the technical strategy of the online identification system. Modeling and prediction are two procedures of the system. In the modeling procedure, the flame features of the known samples are obtained through data acquisition and feature extraction. A model is generated using the whole training data, with a runtime of about $1 \mathrm{~min}$. In the prediction procedure, the flame features of an unknown sample are also obtained through data acquisition and feature extraction. The built model is used to classify the flame features of an unknown sample, and then predict its coal type. The runtime of the prediction procedure is less than $5 \mathrm{~s}$, which meets the requirement of online identification of coal types in industrial applications.

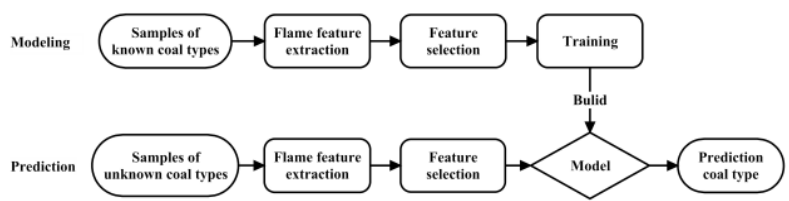

Fig. 5 Technical strategy of the online identification system

\section{Results and discussion}

\subsection{Operating conditions}

Two single coals ( $\mathrm{A}$ and $\mathrm{B}$ ) and three coal blends (3A1B, $\mathrm{AB}$, and $1 \mathrm{~A} 3 \mathrm{~B}$ ) were used to investigate the identification of coal blends. Coals $3 \mathrm{~A} 1 \mathrm{~B}, \mathrm{AB}$, and $1 \mathrm{~A} 3 \mathrm{~B}$ were composed of coal $\mathrm{A}$ and coal $\mathrm{B}$ with mass mixing ratios of $75 \%: 25 \%, 50 \%: 50 \%$, and $25 \%: 75 \%$, respectively. The proximate and ultimate analysis data of the five coal types are presented in Table 2. The tested coals were pulverized and sieved to a particle diameter of 70-160 $\mu \mathrm{m}$ and dried before the experiments. The operating conditions, including variations in coal feed rate, total air flow rate and PA/SA ratio, are presented in Table 3. A detailed description of the operating conditions is presented by Zhou et al. (2014). Each coal type was tested in 12 test cases, resulting in $60(12 \times 5)$ test cases in total. Multiple data acquisition was applied to increase the sample size and reduce experimental errors (e.g., 8 continuous samplings for each test case). As a result, a total of $480(8 \times 60)$ datasets were obtained in the tests.

Table 2 Proximate and ultimate analysis of coals

\begin{tabular}{|c|c|c|c|c|c|}
\hline Sample & A & 3A1B & $\mathrm{AB}$ & $1 \mathrm{~A} 3 \mathrm{~B}$ & B \\
\hline \multicolumn{6}{|c|}{ Proximate analysis (wt\%, ar) } \\
\hline M & 10.69 & 10.09 & 9.49 & 8.9 & 6.95 \\
\hline $\mathrm{V}$ & 28.94 & 27.88 & 26.82 & 25.75 & 29.46 \\
\hline $\mathrm{F}$ & 46.92 & 45.70 & 44.47 & 43.25 & 47.35 \\
\hline A & 13.45 & 16.33 & 19.22 & 22.10 & 16.24 \\
\hline \multicolumn{6}{|c|}{ Ultimate analysis (wt\%, ar) } \\
\hline $\mathrm{C}$ & 62.22 & 60.07 & 57.92 & 55.77 & 62.5 \\
\hline $\mathrm{H}$ & 4.11 & 3.99 & 3.87 & 3.76 & 4.11 \\
\hline $\mathrm{O}$ & 7.80 & 7.85 & 7.89 & 7.94 & 8.05 \\
\hline $\mathrm{N}$ & 0.92 & 0.90 & 0.88 & 0.85 & 0.99 \\
\hline $\mathrm{S}$ & 0.81 & 0.77 & 0.73 & 0.68 & 0.64 \\
\hline$Q_{\text {ar,net }}(\mathrm{MJ} / \mathrm{kg})$ & 23.92 & 23.12 & 22.32 & 21.52 & 23.88 \\
\hline
\end{tabular}

Table 3 Operating conditions

\begin{tabular}{cccc}
\hline Case & $\begin{array}{c}\text { Coal feed rate } \\
(\mathrm{kg} / \mathrm{h})\end{array}$ & $\begin{array}{c}\text { Total air flow } \\
\text { rate }(\mathrm{kg} / \mathrm{h})\end{array}$ & PA/SA ratio \\
\hline 1 & 25 & 210.4 & 0.339 \\
2 & 25 & 227.6 & 0.340 \\
3 & 25 & 245.8 & 0.340 \\
4 & 25 & 210.6 & 0.380 \\
5 & 25 & 227.5 & 0.380 \\
6 & 25 & 245.9 & 0.381 \\
7 & 30 & 252.7 & 0.340 \\
8 & 30 & 273.0 & 0.341 \\
9 & 30 & 294.9 & 0.340 \\
10 & 30 & 252.8 & 0.380 \\
11 & 30 & 273.2 & 0.381 \\
12 & 30 & 295.0 & 0.380 \\
\hline
\end{tabular}

\subsection{Combustion behavior of different coals}

In this study, combustion behavior was used as the basis of coal type identification. To analyze the combustion behavior of coals $\mathrm{A}, 3 \mathrm{~A} 1 \mathrm{~B}, \mathrm{AB}, 1 \mathrm{~A} 3 \mathrm{~B}$, and B, 20 flame features were extracted for each coal type under variable conditions. The ranges of the flame features are shown in Fig. 6 using boxplots, each consisting of $96(8 \times 12)$ data points. The statistical distribution of data for each flame feature is easy to see in the boxplots. The ranges of the spatial flame features of the five coals under variable conditions are shown in Figs. 6a-6b, including the mean and standard deviation of the flame image signal. The ranges of the temporal flame features of the five coals under variable conditions are shown in Figs. 6c-6h, including the mean, standard deviation, skewness, kurtosis, fluctuation ratio, and oscillation frequency of the light intensity signal. There is a high degree of overlap in each flame feature for the five coals. Thus, it is difficult to identify the coal types using traditional methods. There is a need to introduce an SVM method which can use multiple 
flame features in coal identification, considering the advantage of SVM in tackling small samples and

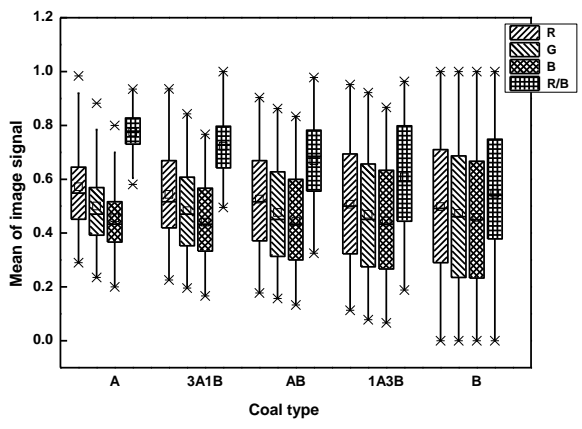

(a)

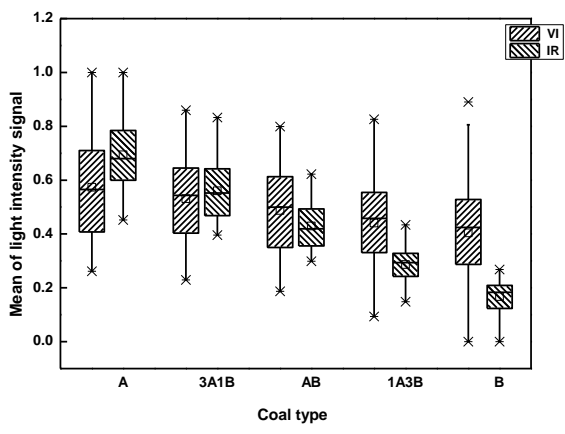

(c)

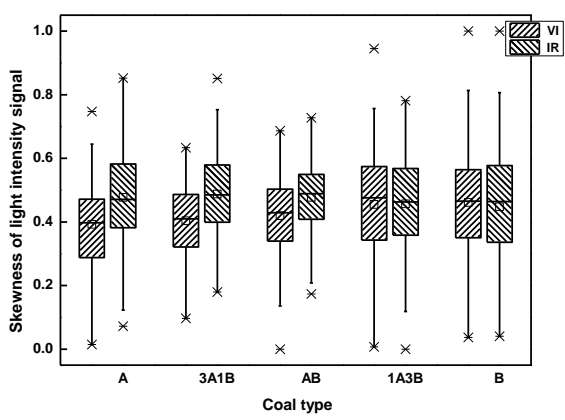

(e)

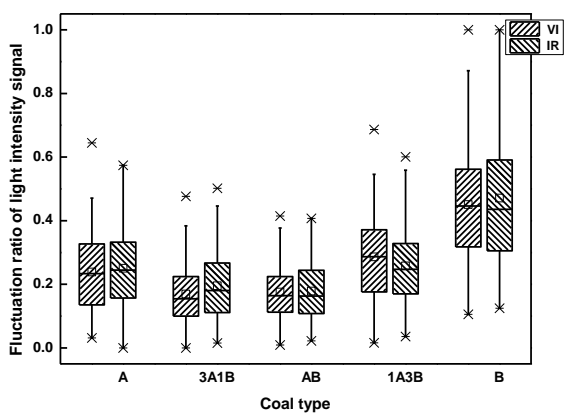

(g) learning problems

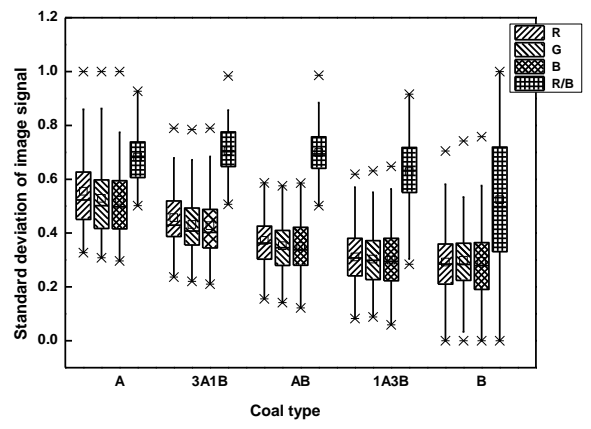

(b)

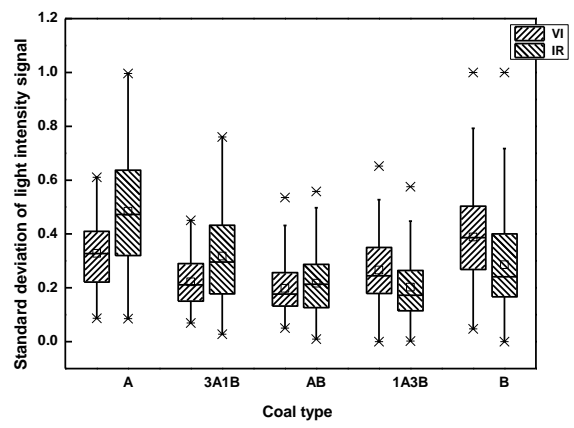

(d)

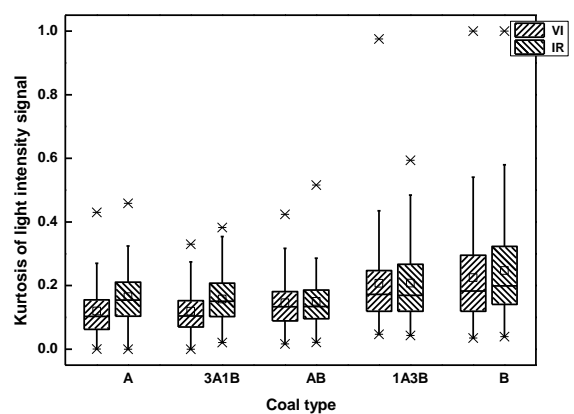

(f)

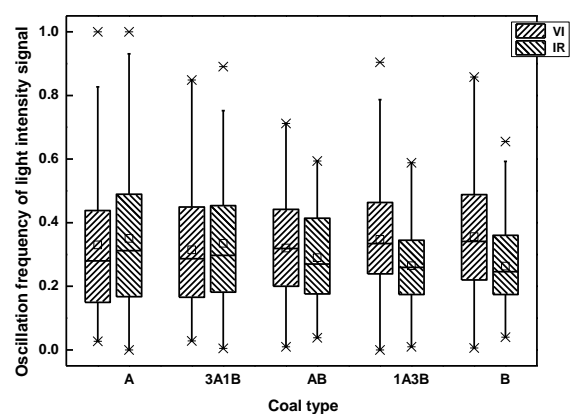

(h)

Fig. 6 Flame features of the five coals: (a)-(b) spatial flame features; (c)-(h) temporal flame features 


\subsection{Importance of different flame features}

Fig. 7a shows the rankings, arranged in descending order, and the importance weights of the 20 flame features. The cumulative importance weight in relation to the number of flame features is shown in Fig. $7 \mathrm{~b}$, with the flame features ranked in descending order. The results show that the importance weight of $S_{\text {mean,IR }}$ is obviously larger than those of other flame features. It can be inferred that $S_{\text {mean,IR }}$ is the most influential feature in coal type identification. The first 18 flame features explain $99.73 \%$ of the information of the datasets. Therefore, these 18 flame features could be selected to identify coal types very well. The remaining 2 flame features have almost no effect on coal type identification. In relation to the temporal features, $P_{\mathrm{std}, \mathrm{R}}, P_{\mathrm{std}, \mathrm{G}}$, and $P_{\mathrm{std}, \mathrm{B}}$ are more influential than $P_{\text {mean, },}, P_{\text {mean, },}$, and $P_{\text {mean, B }}$ in coal type identification, while $P_{\mathrm{std}, \mathrm{R} / \mathrm{G}}$ had a similar effect to

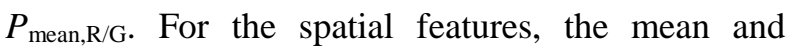
standard deviation of the IR signal were clearly more significant than those of the VI signal.

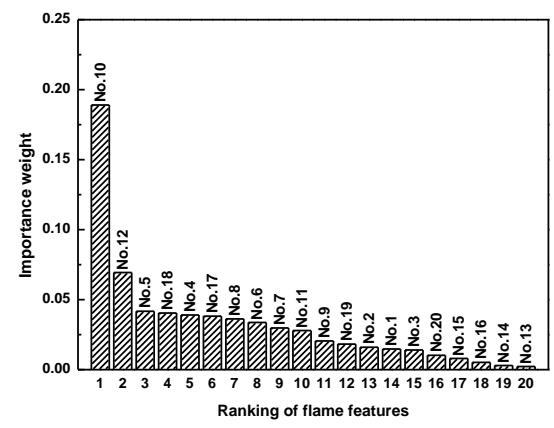

(a)

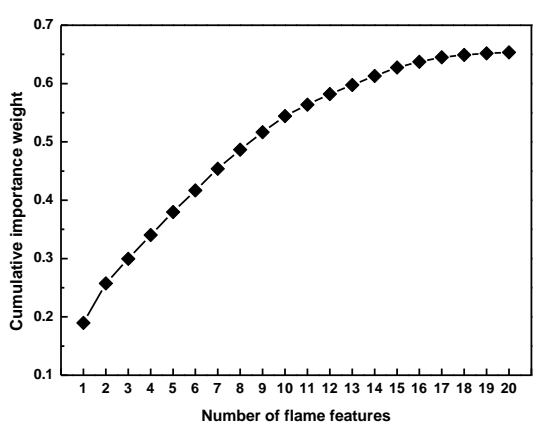

(b)

Fig. 7 Importance and cumulative importance weights: (a) importance weight and ranking of each flame feature; (b) cumulative importance weight in relation to the number of flame features

In addition, the runtime of ReliefF in analyzing the importance of each flame feature was less than that of the single flame feature modeling method (Zhou et al., 2014).

\subsection{Effects of the number of flame features on identification}

Samples of coals A, 3A1B, AB, 1A3B and B were tested to study coal type identification. All 480 datasets of the five coals were randomly divided into two groups: a training group and a test group, processing $75 \%$ and $25 \%$ of the datasets of each coal type, respectively. The training group (360 datasets) was used to generate an SVM model. The number of samples was 6 (over 5) in each case for each coal type to ensure model accuracy (Zhou et al., 2014). The testing group (120 datasets) was used to evaluate the success rate of coal type identification which was calculated as

$$
R_{\text {success }}=N_{\text {success }} / N_{\text {total }},
$$

where $R_{\text {success }}$ is the success rate, $N_{\text {success }}$ is the number of successful identifications, and $N_{\text {total }}$ is the total number of identifications.

The method for selecting an optimal number of features for use in the SVM was to compare the success rates of different numbers of flame features. SVM models were created by the training group using different numbers of flame features, and the test group was used to evaluate their performances. Flame features were ranked by their importance weights, from the largest to the smallest. Feature vectors, consisting of different numbers of flame features, were tested and compared to select an optimal feature vector. Each grouping-training-testing procedure was carried out 20 times.

Fig. 8 shows the mean success rates of identification of the five coals in different numbers of flame features. Each data point in Fig. 8 is an average of $100(5 \times 20)$ success rates, and the standard deviation of the data is shown as an error bar. The mean success rate increased as the number of flame features increased when the number of flame features was less than 12. However, it showed no further significant increase when the number of flame features was more than 12. Computational 
complexity increased quickly as the number of flame features increased. In addition, the error bar remained basically stable when the number of flame features was over 12. Considering the overall precision, efficiency and stability, the first 12 of the ranked flame features were selected to identify the coal types in this study. The selected flame features were Nos. $10,12,5,18,4,17,8,6,7,11,9$ and 19 (Table 1).

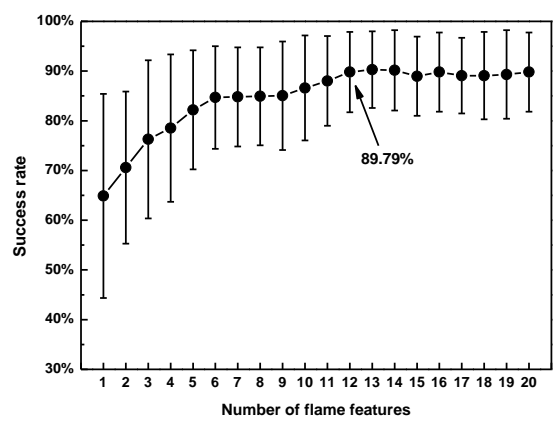

Fig. 8 Success rate changes with the number of flame features

\subsection{Effects of similarity on identification}

A coal blend is blended by mixing two single coals in a specific ratio, thus the combustion behavior of a coal blend and its component coals is similar. In consideration of the various importance weights of different flame features, the weighted average similarity coefficient between two coal types was calculated using the importance weights (Section 4.3) as the weighting factors. The first 12 of the ranked flame features (Section 4.3) were selected. Table 4 shows the weighted average similarity coefficient between two different coal types, and the mean of the weighted average similarity coefficients between one coal and the other four coals. $\mathrm{Cs}_{\mathrm{AB}-\mathrm{A} \text {,average }}$ was larger

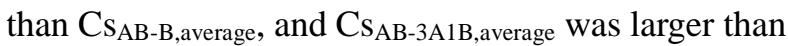
$\mathrm{Cs}_{\mathrm{AB}-1 \mathrm{~A} \text { 3B,average. The combustion behavior of a coal }}$ blend is more similar to that of its component coal of higher volatile matter in the blend. Moreover, the similarity between a coal blend and its component coal increased as the proportion of the component coal increased. These results support those of Chi et al. (2010).

To analyze the effects of the similarity coefficient on coal type identification, the training-testing process was carried out 20 times using the first 12 of the ranked flame features (Section 4.3) for coals $\mathrm{A}, 3 \mathrm{~A} 1 \mathrm{~B}, \mathrm{AB}, 1 \mathrm{~A} 3 \mathrm{~B}$, and $\mathrm{B}$. The numbers of correct identifications and erroneous identifications are presented in Table 5. Since the process was carried out 20 times and the testing group comprised one quarter of the datasets, the total number of identifications was $480(24 \times 20)$ for each coal type. A single coal may be mis-identified as a coal blend, if one component of the blend is predominant, due to the high similarity between them (e.g., coal A was mis-identified as coal 3A1B, and coal B was mis-identified as coal 1A3B). Similarly, a coal blend may be mis-identified as another coal blend if two the blends consist of the same coals in different proportions (e.g., coal 3A1B was mis-identified as coal $\mathrm{AB}$, coal $1 \mathrm{~A} 3 \mathrm{~B}$ was mis-identified as coal $\mathrm{AB}$, and coal $\mathrm{AB}$ was mis-identified as coals $3 \mathrm{~A} 1 \mathrm{~B}$ and $1 \mathrm{~A} 3 \mathrm{~B})$. Also, a coal blend may be mis-identified as a single coal which is a component of the coal blend (e.g., coal 3A1B was mis-identified as coal $\mathrm{A}$, and coal $1 \mathrm{~A} 3 \mathrm{~B}$ was mis-identified as coal B). Thus, in the process of coal type identification, one coal type can be mis-identified as another due to their similar combustion behavior.

The relationship between the error rate and the similarity coefficient was investigated. The error rate was calculated as

$$
R_{\text {error, } \alpha-\beta}=N_{\text {error, } \alpha-\beta} / N_{\text {total }},
$$

where $R_{\text {error }, \alpha-\beta}$ is the error rate of coal $\alpha$ mis-identified as coal $\beta, N_{\text {error }, \alpha-\beta}$ is the number of mis-identifications of coal $\alpha$ as coal $\beta$, and $N_{\text {total }}$ is the total number of identifications.

Table 4 Weighted average similarity coefficients

\begin{tabular}{ccccccc}
\hline \multirow{2}{*}{ Coal $\alpha$} & \multicolumn{5}{c}{ Coal $\beta$} & \multirow{2}{*}{ Mean } \\
\cline { 2 - 6 } & $\mathrm{A}$ & 3A1B & $\mathrm{AB}$ & 1A3B & $\mathrm{B}$ & \\
\hline A & - & 0.6873 & 0.4518 & 0.4003 & 0.3751 & 0.4786 \\
3A1B & 0.6873 & - & 0.6332 & 0.4638 & 0.3904 & 0.5437 \\
AB & 0.4518 & 0.6332 & - & 0.6114 & 0.3613 & 0.5144 \\
1A3B & 0.4003 & 0.4638 & 0.6114 & - & 0.5437 & 0.5048
\end{tabular}


Table 5 Number of correct and erroneous identifications for 20 trials

\begin{tabular}{cccccc}
\multicolumn{6}{c}{ for $\mathbf{2 0}$ trials } \\
\hline \multirow{2}{*}{ Target } & \multicolumn{5}{c}{ Predicting result } \\
\cline { 2 - 6 } & $\mathrm{A}$ & $3 \mathrm{~A} 1 \mathrm{~B}$ & $\mathrm{AB}$ & $1 \mathrm{~A} 3 \mathrm{~B}$ & $\mathrm{~B}$ \\
\hline $\mathrm{A}$ & 450 & 30 & 0 & 0 & 0 \\
$3 \mathrm{~A} 1 \mathrm{~B}$ & 33 & 388 & 59 & 0 & 0 \\
$\mathrm{AB}$ & 0 & 41 & 409 & 30 & 0 \\
1A3B & 0 & 0 & 28 & 441 & 11 \\
$\mathrm{~B}$ & 0 & 0 & 0 & 8 & 472 \\
\hline
\end{tabular}

The error rates of different similarity coefficients are shown Fig. 9. In accordance with the data distribution, segmented linear regression analysis was used to analyze the correlation between the error rate and the similarity coefficient. The correlation coefficients of the two fitted lines were $1\left(r^{2}=1\right)$ and $0.8103\left(r^{2}=0.6566\right)$, respectively. The dividing point is the intersection point of the two fitted lines. The abscissa value of the point was 0.4709 . The error rate was zero when the similarity coefficient was less than 0.4709. This indicates that a coal type would not be mis-identified as another coal type if the similarity coefficient between the two coal types was below 0.4709 . When the similarity coefficient was more than 0.4709 , the error rate had a positive correlation with the similarity coefficient. While the value of the dividing point was 0.4709 in the current study, the value may change for other coal types.

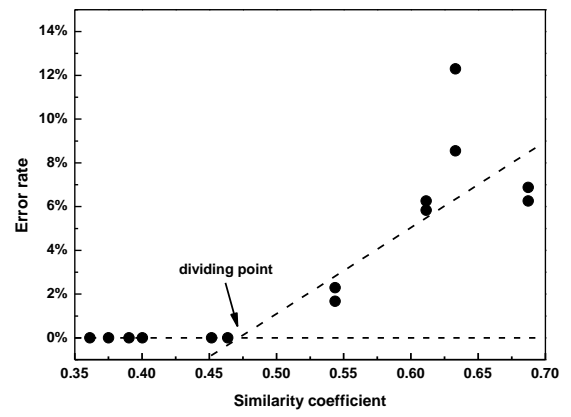

Fig. 9 Error rates for different similarity coefficients

The relationship between the success rate and the similarity coefficient was also investigated. The mean similarity coefficient was calculated as the mean of the weighted average similarity coefficients between one coal and the other four coals. The success rate decreased as the mean similarity coefficient increased (Fig. 10). A higher similarity coefficient resulted in a lower success rate. Because coal 3A1B had the highest mean similarity coefficient with the other coals, it, mis-identified as coals A and $\mathrm{AB}$ possibly, had the lowest success rate among the five coals. In contrast, coal B had the highest success rate, because it had the lowest mean similarity coefficient with the other coals.

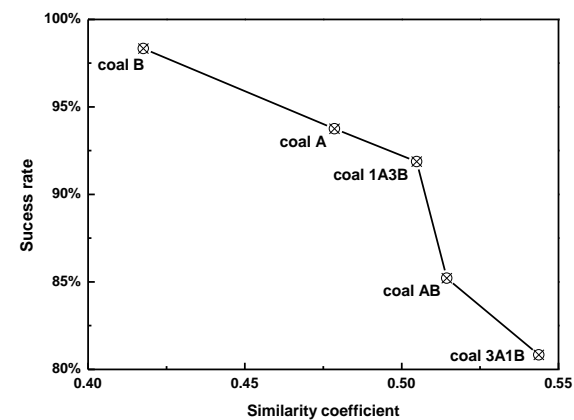

Fig. 10 Success rate changes with the mean similarity coefficient

\section{Conclusions}

In this study, tests were conducted on a $300 \mathrm{~kW}$ combustion test facility under variable conditions. Twenty flame features were extracted to investigate the combustion behavior of different coal types. The method of ReliefF was used to calculate the importance weight of each flame feature quantitatively, with a very short runtime. $S_{\text {mean,IR was }}$ the most important of the 20 flame features, and played the dominant role in coal type identification. The number of flame features used to build an SVM model was reduced from 20 to 12 through combining the methods of ReliefF and SVM. The computational complexity was reduced, and the computational precision was guaranteed. The optimal flame features were $S_{\text {mean,IR }}$ (No. 10), $S_{\text {std,IR }}$ (No. 12), $P_{\text {std,R }}$ (No. 5), $S_{\text {flu,IR }}$ (No. 18), $P_{\text {mean }, \mathrm{R} / \mathrm{G}}$ (No. 4), $S_{\mathrm{flu}, \mathrm{VI}}$ (No. 17), $P_{\text {std,R/G }}\left(\right.$ No. 8), $P_{\text {std,G }}$ (No. 6), $P_{\text {std,B }}$ (No. 7) $S_{\text {std,VI }}($ No. 11), $S_{\text {mean,VI }}$ (No. 9), and $S_{f, \mathrm{VI}}$ (No. 19). The defined similarity coefficient was able to analyze the similarity of combustion behavior between different coal types. The similarity coefficient between a coal blend and its component was influenced mainly by the volatile matter in the coal blend and its mixing 
ratio. A coal blend had a higher similarity coefficient with its component of higher volatile matter and mixing ratio in the blend. A threshold value of 0.4709 was found for the relationship between the error rate and the similarity coefficient. The error rate remained at zero when the similarity coefficient was below the threshold. When the similarity coefficient was above the threshold, the error rate generally increased as the similarity coefficient increased. In addition, the success rate decreased as the similarity coefficient increased. A higher similarity coefficient resulted in a lower success rate. It was more difficult to identify a coal type that had a high similarity with other coal types, due to the high error rates. The present results demonstrate that the proposed system is effective for identifying coal blends with similar combustion behavior.

\section{References}

Ballester, J., García-Armingol, T., 2010. Diagnostic techniques for the monitoring and control of practical flames, Progress in Energy and Combustion Science. 36(4):375-411. [doi:10.1016/j.pecs.2009.11.005]

Biswas, S., Choudhury, N., Sarkar, P., Mukherjee, A., Sahu, S.G., Boral, P., Choudhury, A., 2006. Studies on the combustion behaviour of blends of indian coals by tga and drop tube furnace, Fuel Processing Technology. 87(3):191-199. [doi:10.1016/j.fuproc.2005.05.002]

Chang, C.C., Lin, C.J., 2011. Libsvm: A library for support vector machines, Acm Transactions on Intelligent Systems and Technology. 2(3): 27. [doi:10.1145/1961189.1961199]

Chi, T., Zhang, H., Yan, Y., Zhou, H., Zheng, H., 2010. Investigations into the ignition behaviors of pulverized coals and coal blends in a drop tube furnace using flame monitoring techniques, Fuel. 89(3):743-751. [doi:10.1016/j.fuel.2009.06.010]

Cloke, M., Lester, E., Thompson, A.W., 2002. Combustion characteristics of coals using a drop-tube furnace, Fuel. 81(6):727-735. [doi:10.1016/S0016-2361(01)00199-5]

Cortes, C., Vapnik, V., 1995. Support-vector networks, Machine Learning. 20(3):273-297.
[doi:10.1023/A:1022627411411]

Haas, J., Tamura, M., Weber, R., 2001. Characterisation of coal blends for pulverised fuel combustion, Fuel. 80(9):1317-1323. [doi:10.1016/S0016-2361(00)00216-7]

Hsu, C.W., Lin, C.J., 2002. A comparison of methods for multiclass support vector machines, IEEE Transactions on Neural Networks. 13(2):415-425. [doi:10.1109/72.991427]

Huang, B.Y., Luo, Z.X., Zhou, H.C., 2010. Optimization of combustion based on introducing radiant energy signal in pulverized coal-fired boiler, Fuel Processing Technology. 91(6):660-668. [doi:10.1016/j.fuproc.2010.01.015]

Huang, H.-W., Zhang, Y., 2008. Flame colour characterization in the visible and infrared spectrum using a digital camera and image processing, Measurement Science and Technology. 19(8):085406. [doi:10.1088/0957-0233/19/8/085406]

Huang, Y., Yan, Y., 2000. Transient two-dimensional temperature measurement of open flames by dual-spectral image analysis, Transactions of the Institute of Measurement and Control. 22(5):371-384. [doi:10.1177/014233120002200503]

Huang, Y., Yan, Y., Riley, G., 2000. Vision-based measurement of temperature distribution in a $500-\mathrm{kw}$ model furnace using the two-colour method, Measurement. 28(3):175-183. [doi:10.1016/S0263-2241(00)00010-5]

Jiang, Z.-W., Luo, Z.-X., Zhou, H.-C., 2009. A simple measurement method of temperature and emissivity of coal-fired flames from visible radiation image and its application in a $\mathrm{cfb}$ boiler furnace, Fuel. 88(6):980-987. [doi:10.1016/j.fuel.2008.12.014]

Kononenko, I., Simec, E., Robniksikonja, M., 1997. Overcoming the myopia of inductive learning algorithms with relieff, Applied Intelligence.

7(1):39-55. [doi:10.1023/A:1008280620621]

Li, X.L., Wu, M.J., Lu, G., Yan, Y., Liu, S., 2015. On-line identification of biomass fuels based on flame radical imaging and application of radical basis function neural network 
techniques, Iet Renewable Power Generation. $\quad 9(4): 323-330$. [doi:10.1049/iet-rpg.2013.0392]

Lu, G., Yan, Y., Colechin, M., Hill, R., 2006. Monitoring of oscillatory characteristics of pulverized coal flames through image processing and spectral analysis, Ieee Transactions on Instrumentation and Measurement. $\quad$ 55(1):226-231. [doi:10.1109/Tim.2005.861254]

Molcan, P., Lu, G., Bris, T.L., Yan, Y., Taupin, B., Caillat, S., 2009. Characterisation of biomass and coal co-firing on a 3 mwth combustion test facility using flame imaging and gas/ash sampling techniques, Fuel. 88(12):2328-2334.

[doi:10.1016/j.fuel.2009.06.027]

Moon, C., Sung, Y., Ahn, S., Kim, T., Choi, G., Kim, D., 2013. Thermochemical and combustion behaviors of coals of different ranks and their blends for pulverized-coal combustion, Applied Thermal Engineering. 54(1):111-119. [doi:10.1016/j.applthermaleng.2013.01.009]

Osorio, E., Ghiggi, M.L.F., Vilela, A.C.F., Kalkreuth, W.D., Borrego, A.G., 2008. Non-isothermal combustion behaviour of coal blends in a thermobalance as seen by optical microscopy, Thermochimica Acta. 475(1-2):1-7. [doi:10.1016/j.tca.2008.06.002]

Peralta, D., Paterson, N.P., Dugwell, D.R., Kandiyoti, R., 2001. Coal blend performance during pulverised-fuel combustion: Estimation of relative reactivities by a bomb-calorimeter test, $\quad$ Fuel. $\quad \mathbf{8 0}(11)$ :1623-1634. [doi:10.1016/S0016-2361(01)00031-X]

Piramuthu, S., 2003. On learning to predict web traffic, Decision Support Systems. 35(2):213-229. [doi:10.1016/S0167-9236(02)00107-0]

Qiu, T., Yan, Y., Lu, G., 2012. An autoadaptive edge-detection algorithm for flame and fire image processing, Ieee Transactions on Instrumentation and Measurement. 61(5):1486-1493. [doi:10.1109/Tim.2011.2175833]

Sarkar, P., Mukherjee, A., Sahu, S.G., Choudhury, A.,
Adak, A.K., Kumar, M., Choudhury, N., Biswas, S., 2013. Evaluation of combustion characteristics in thermogravimetric analyzer and drop tube furnace for indian coal blends, Applied Thermal Engineering. 60(1-2):145-151.

[doi:10.1016/j.applthermaleng.2013.06.054]

Sun, D., Lu, G., Zhou, H., Yan, Y., 2011. Flame stability monitoring and characterization through digital imaging and spectral analysis, Measurement Science and Technology. 22(11): 114007. [doi:10.1088/0957-0233/22/11/114007]

Tan, C., Xu, L.J., Li, X.M., Yan, Y., 2012. Independent component analysis-based fuel type identification for coal-fired power plants, Combustion Science and Technology. 184(3):277-292.

[doi:10.1080/00102202.2011.635613]

Wang, F., Wang, X., Ma, Z., Yan, J., Chi, Y., Wei, C., Ni, M., Cen, K., 2002. The research on the estimation for the nox emissive concentration of the pulverized coal boiler by the flame image processing technique, Fuel. 81(16):2113-2120. [doi:10.1016/s0016-2361(02)00145-x]

Xu, L.J., Yan, Y., Cornwell, S., Riley, G., 2005. Online fuel tracking by combining principal component analysis and neural network techniques, Ieee Transactions on Instrumentation and Measurement. 54(4):1640-1645. [doi:10.1109/Tim.2005.851203]

Zhou H, Li L.T., Zhang H.L., Zhou B., 2015, Research on the slagging characteristics of blended coals in a pilot-scale furnace, Journal of Zhejiang University SCIENCE A. 16(3): 204-216. [doi:10.1631/jzus.A1400172]

Zhou, H., Tang, Q., Yang, L.B., Yan, Y., Lu, G., Cen, K.F., 2014. Support vector machine based online coal identification through advanced flame monitoring, Fuel. 117:944-951. [doi:10.1016/j.fuel.2013.10.041] 
题 目 : 结合火焰监测技术和支持向量机算法的混煤在线 辨识研究

目 的 : 混煤在锅炉燃烧中的广泛应用。利用火焰监测技 术提取混煤燃烧的火焰特征量, 并获取最优的特 征量组合。研究混煤相似度对其辨识错误率和正 确率的影响。

创新点 : 1. 利用 ReliefF 算法和 SVM 算法定量分析各个火 焰特征量在煤质辨识过程中的重要性, 获取最优 特征量组合。2. 定义混煤/单煤的相似度, 并分析 相似性对其辨识错误和正确率的影响。

方 法: 1. 利用火焰监测技术提取火焰图像信号和火焰光 强信号, 提取 20 个火焰特征量（图 3、图 4 和表 1)；2.利用 ReliefF 算法计算 20 个特征量在煤质 辨识中的重要性 (图 7); 3. 利用 SVM 算法分析 特征量数量对煤质辨识正确率的影响, 确定最优 特征量组合 (图 8)。

结 论 : 1. 在煤质辨识过程中, 结合 ReliefF 算法和 SVM 算法可以将特征量数量由 20 降至 12 , 并保证辨 识准确度；2. 混煤与其组分单煤的相似度主要受 单煤挥发份含量及掺混比例影响; 3. 辨识错误率 与相似度之间存在一个阈值, 当相似度低于该阈 值时, 辨识错误率为 0 , 当相似度高于该阈值时 辨识错误率与相似度呈正相关; 4. 辨识正确率随 着相似度的升高而降低。

关键词 : 混煤; 火焰监测; 在线辨识; ReliefF; SVM; 相 似度 